\title{
Le paléolithique inférieur et moyen en Midi toulousain : nouvelles données et perspectives de l'archéologie préventive
}

Early and Middle Paleolithic sites in the Midi Toulousain Region: new data and research-orientations in preventive archeology

L. Bruxelles, A.-L. Berthet, P. Chalard, D. Colonge, G. Delfour, M. Jarry, L.-A. Lelouvier, T. Arnoux et 0 . Onézime

\section{OpenEdition}

Journals

Édition électronique

URL : http://journals.openedition.org/paleo/1193

DOI : $10.4000 /$ paleo. 1193

ISSN : 2101-0420

Éditeur

SAMRA

Édition imprimée

Date de publication : 1 décembre 2003

Pagination : 7-28

ISSN : 1145-3370

Référence électronique

L. Bruxelles, A.-L. Berthet, P. Chalard, D. Colonge, G. Delfour, M. Jarry, L.-A. Lelouvier, T. Arnoux et O. Onézime, «Le paléolithique inférieur et moyen en Midi toulousain : nouvelles données et perspectives de l'archéologie préventive », PALEO [En ligne], 15 | 2003, mis en ligne le 02 août 2010, consulté le 07 juillet 2020. URL : http://journals.openedition.org/paleo/1193 ; DOI : https://doi.org/10.4000/paleo. 1193

Ce document a été généré automatiquement le 7 juillet 2020.

\section{cc) (†)}

PALEO est mis à disposition selon les termes de la licence Creative Commons Attribution - Pas d'Utilisation Commerciale - Pas de Modification 4.0 International. 


\title{
Le paléolithique inférieur et moyen en Midi toulousain : nouvelles données et perspectives de l'archéologie préventive
}

\author{
Early and Middle Paleolithic sites in the Midi Toulousain Region: new data and \\ research-orientations in preventive archeology
}

L. Bruxelles, A.-L. Berthet, P. Chalard, D. Colonge, G. Delfour, M. Jarry, L.-A. Lelouvier, $\mathrm{T}$. Arnoux et $\mathrm{O}$. Onézime

\section{1 - Introduction}

1 Le bassin de la Garonne en Midi toulousain est connu depuis le début du siècle dernier pour sa richesse en industries lithiques attribuables principalement au Paléolithique inférieur et moyen (Breuil 1937). Ces découvertes ont été effectuées systématiquement sur les basse et moyenne terrasses du fleuve, principalement lors de prospections de surface. Dans de rares cas, à la faveur de travaux d'aménagement ou d'exploitation (Méroc 1961), les premières observations stratigraphiques ont pu être effectuées dans des coupes démontrant la position exacte des vestiges acheuléens au sein de la moyenne terrasse. En rive droite de l'Ariège, principal affluent de la Garonne dans ce secteur, seules les fouilles menées à l'Infernet sur la commune de Clermont-le-Fort (Méroc et Paloumé 1958) offraient des résultats appréciables, bien que la faune et les industries lithiques décrites aient été remaniées dans les alluvions d'un ruisseau.

Ce potentiel archéologique, appréhendé très tôt au travers de l'abondance des vestiges et s'inscrivant certes dans un cadre géomorphologique vaste (Cavaillé 1965; Hubschman 1975a et b) demandait pourtant une révision indispensable des données récoltées depuis ces premières études. En effet, la multiplication des opérations archéologiques programmées ou préventives (prospections, sondages et évaluations, fouilles) a considérablement bouleversé notre connaissance des contextes 
géomorphologiques et sédimentologiques susceptibles de receler ces industries paléolithiques. Par ailleurs, des hypothèses quant à l'évolution des productions lithiques au cours de la période acheuléenne et de son évolution vers le Paléolithique moyen peuvent être également avancées. Cet article se propose donc de présenter un état des travaux dans ce domaine et d'offrir des perspectives de recherches prometteuses, s'appuyant notamment sur les toutes dernières investigations menées par l'Institut National de Recherches Archéologiques Préventives (INRAP) dans le Midi Toulousain.

\section{2 - Le Midi toulousain}

\section{1 - Cadre géographique et géomorphologique}

Située au sud-est du bassin aquitain, la région du Midi toulousain se compose de deux grandes unités géomorphologiques : la vallée de la Garonne et la molasse tertiaire qui affleurent de part et d'autre de cette dernière. Débutant au pied des premiers reliefs de la zone sous-pyrénéenne, elle est limitée, dans sa partie est, par des lignes de cuestas qui assurent la transition avec les massifs de la Montagne Noire. L'ensemble du territoire présente fréquemment des formations superficielles limoneuses déposées au cours du Quaternaire (Icole et Rieucau 1982a).

Figure 1 - Contexte géomorphologique du Midi toulousain avec localisation des sites présentés (dessin L. Bruxelles/INRAP).

Figure 1 - Geomorphological context of Midi toulousain region and location of the studied palaeolithic sites.

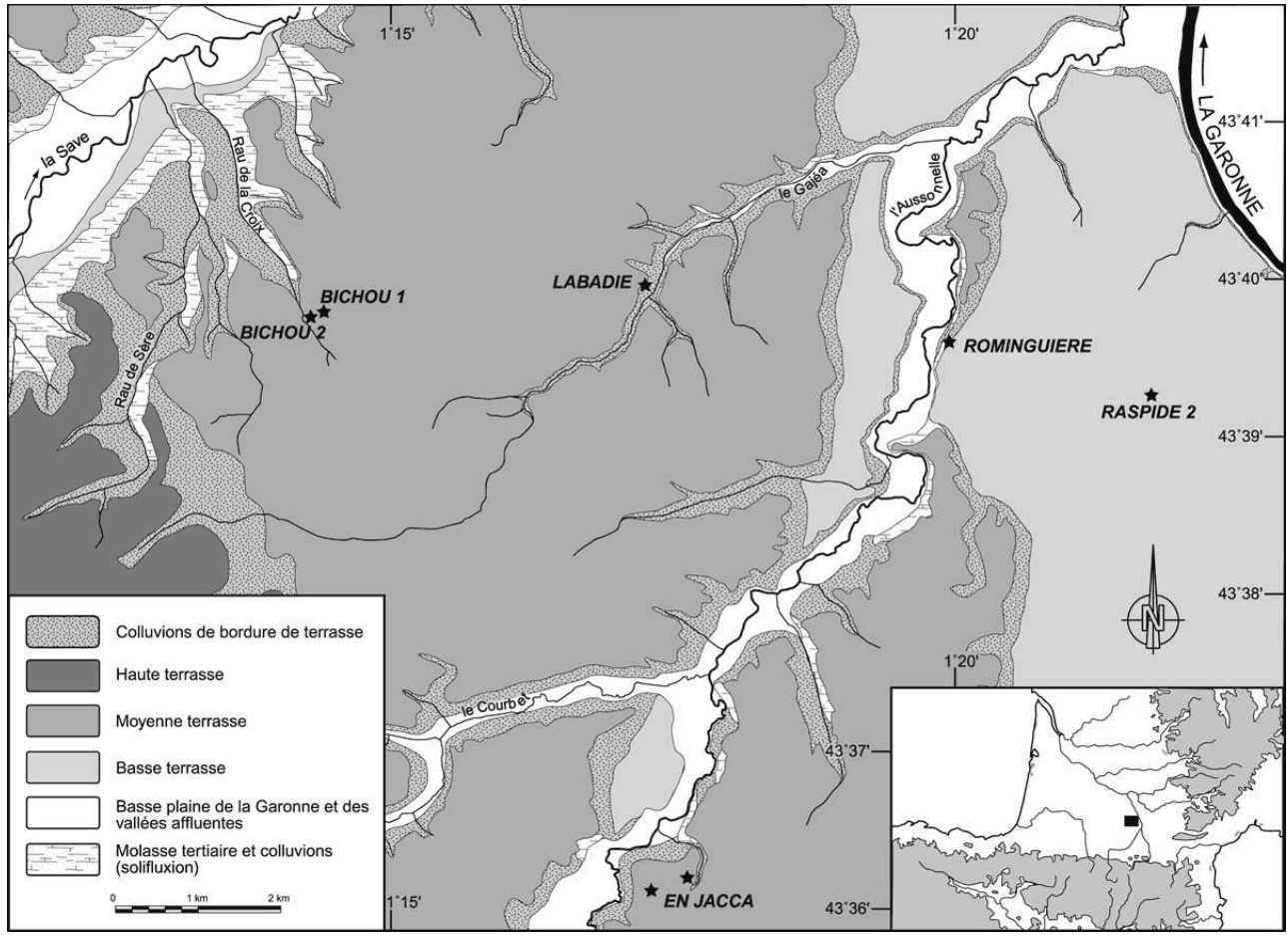




\section{La molasse tertiaire}

4 D'origine pyrénéenne, la molasse présente dans le secteur étudié des faciès argilocalcaires qui portent le nom de "Terrefort". Ces matériaux meubles conditionnent le paysage qui borde la vallée de la Garonne : le Lauragais à l'est et le Savès gascon à l'ouest qui se caractérisent par des coteaux à pentes douces découpés dans les molasses oligocène et miocène. En revanche, dans le secteur sud de la vallée de la Garonne, l'apparition de faciès plus résistants offre un relief accusé dans les pays du BasComminges et du Volvestre (Hubschman 2000). L'érosion des coteaux molassiques, dès la fin du Tertiaire, a provoqué un rajeunissement des reliefs de plusieurs dizaines de mètres (Hubschman 1975a).

\section{La vallée de la Garonne}

5 Le creusement de la vallée de la Garonne a donné lieu à la formation d'un grand axe morphologique qui entame les dépôts molassiques depuis les Pyrénées en direction du nord. C'est en Midi toulousain que la vallée présente son ampleur maximale, notamment au niveau de la ville de Toulouse où sa largeur avoisine $25 \mathrm{~km}$ d'est en ouest. C'est également à cet endroit que son profil dissymétrique est le plus manifeste. En effet, la rive orientale, taillée dans la molasse, est très abrupte. Elle s'oppose à un vaste système de terrasses étagées (fig. 1) dont la mise en place débute à la fin du Tertiaire et concerne la totalité du Quaternaire. L'analyse pétrographique témoigne de l'origine pyrénéenne des matériaux caillouteux (Hubschman 1975b). Cinq épisodes d'alluvionnement se détectent dans le paysage, formant des paliers successifs bien marqués dans le secteur toulousain (Hubschman 1975b, 1975c, 1975d, 2000 ; Icole et Rieucau 1982b) :

- la nappe culminante : c'est une formation complexe qui surplombe la Garonne de 150 à 200 $\mathrm{m}$. Elle domine également l'ensemble du paysage molassique. Son aspect est très dégradé et seuls subsistent quelques lambeaux entaillés par de nombreuses vallées secondaires ;

- la haute terrasse est également fortement affectée par l'érosion. Mieux préservée dans sa partie amont, elle forme des plateaux discontinus, tels que ceux de Rieumes et de la forêt de Bouconne. Ces derniers dominent la Garonne de $90 \mathrm{~m}$;

- la moyenne terrasse : limitée à l'est par un talus de $30 \mathrm{~m}$, elle se remarque par sa continuité et son ampleur. Bien individualisée dans le paysage, elle se place à des altitudes oscillant entre 50 et $80 \mathrm{~m}$ au-dessus de la Garonne, formant localement des paliers successifs. L'épaisseur des alluvions, variable, atteint $14 \mathrm{~m}$ près de Colomiers à l'ouest de Toulouse (Capdeville et al. 1997) ;

- la basse terrasse est particulièrement bien conservée dans la vallée de la Garonne. Son altitude varie de l'amont vers l'aval entre 35 et $20 \mathrm{~m}$ au dessus du cours actuel de la Garonne ;

- la basse plaine : séparée par un talus de 5 à $10 \mathrm{~m}$ de la basse terrasse, ses alluvions sont parfois difficiles à distinguer des faciès du lit majeur postglaciaire et sub-actuel. Elle se place à environ $15 \mathrm{~m}$ au-dessus de l'étiage de la Garonne.

Notons qu'il a pu être démontré, grâce à l'étude comparative des sols et des phénomènes d'altération des éléments grossiers, qu'il existe un décalage chronologique dans la mise en place de ces dépôts entre les secteurs amont et aval de la vallée. Cependant, cette hypothèse n'est pas suffisamment étayée pour être admissible pour 
les périodes anciennes des moyenne et haute terrasses (Bourgeat et al. 1984 ; Icole 1973 et 1980).

\section{Les formations superficielles}

Elles sont d'origines diverses et se sont toutes déposées au cours du Quaternaire. Elles rassemblent :

- les colluvions de l'avant-pays molassique. Les versants du Terrefort sont en effet couramment dissimulés sous des limons colluviaux résultant du démantèlement des plateaux molassiques. Ils se sont mis en place durant la fin de la dernière glaciation ou au cours des premières phases du Postglaciaire (Hubschman 1975a);

- les lœess, surtout représentés dans la vallée de l'Hers mort à Pompertuzat, rapportables au dernier maximum glaciaire, ont été datés de $20900 \pm 570$ ans B.P. (14C réalisé sur des coquilles de gastéropodes) (Hubschman 1975a). Ils se retrouvent aussi localement au bas des talus de la basse terrasse et de la basse plaine de la vallée de la Garonne;

- les dépôts de pente recouvrent les talus délimitant les terrasses alluviales et sur les grands versants des vallées dissymétriques. L'érosion actuelle participe encore très activement au rajeunissement des reliefs (Icole et Rieucau 1982a);

- les couvertures limoneuses, d'épaisseur variable, déposées sur les nappes alluviales du bassin de la Garonne. Les questions concernant leur mise en place sont abordées dans le paragraphe suivant.

\section{2 - Historique des recherches géologiques}

8 De nombreux auteurs, géologues et préhistoriens, ont travaillé sur les terrasses de la Garonne et les autres vallées secondaires du bassin garonnais. Les travaux de J. Hubschman (1975a, 1975b, 1975c, 1975d) ont largement contribué à la connaissance du système alluvial garonnais. Ainsi, Ils montrent que chaque remblaiement alluvial développe une évolution pédologique propre (Hubschman 1975b, 1975c; Icole et Rieucau 1982b) :

- les dépôts de la basse plaine se caractérisent le plus souvent par des profils bien différenciés de sols bruns lessivés accompagnés d'une illuviation peu marquée. Le matériel graveleux est peu altéré ;

- la basse terrasse présente en revanche des profils et un degré d'altération qui contrastent avec ceux de la basse plaine. Les sols lessivés hydromorphes s'y développent avec parfois la formation d'un horizon d'accumulation du fer et du manganèse reconnu sous le terme de " grep ». Les alluvions grossières montrent une altération des galets cristallins plus poussée, une matrice plus colorée et un faciès argilique net ;

- les remblaiements antérieurs ont également développé des profils type lessivé hydromorphe. La moyenne terrasse se distingue nettement de la basse terrasse car elle constitue la première nappe offrant des faciès franchement altérés, argilisés, colmatés et rubéfiés (l'horizon de « grep » est bien marqué);

- la haute terrasse ainsi que la nappe culminante montrent des évolutions similaires à celle de la moyenne terrasse. Cependant, elles s'en différencient par un degré d'altération encore plus poussé qui affectent les dépôts sur des profondeurs plus importantes, proportionnelles à l'ancienneté des alluvions.

9 Le problème de la chronologie, de l'origine et du mode de mise en place des couvertures limoneuses des terrasses, qui bien souvent scellent les niveaux 
archéologiques, demeure d'actualité. Trois principales hypothèses s'opposent. Les arguments d'ordre sédimentologiques, pédologiques et géochimiques amènent les différents auteurs à considérer cette couverture superficielle :

- soit d'origine fluviatile de fin de crue, donc contemporaine de chaque épisode

d'alluvionnement (Hubschman 1975c) ;

- soit constituée d'apports essentiellement éoliens par saupoudrage (Dupuis, 1958 ; Enjalbert

1960 ; Alimen 1964 ; Rieucau 1975 cités In : Icole et Rieucau 1982a) ;

- soit issue de remaniements postérieurs in situ (haute et moyenne terrasses) à partir du matériel prélevé sur les nappes alluviales (Icole 1973).

Pour M. Icole et L. Rieucau (1982a), les indices chronologiques apportés principalement par leur contenu archéologique, supposent que les limons sont plus jeunes que les nappes qui les supportent et dans tous les cas, postérieurs à la mise en place de la moyenne terrasse. Les derniers travaux, réalisés notamment sur les moyenne et basse terrasses, vont aussi dans le sens d'apports limoneux relativement récents (Capdeville et al. 1997 ; Millet 2001).

Malgré les différentes approches méthodologiques, la complexité des phénomènes post-sédimentaires qui se sont exercés sur les terrasses graveleuses et leur couverture limoneuse ont souvent abouti à des conclusions contradictoires (Hubschman et al. 1980). Par contre, ces critères pédogéochimiques et d'altération permettent de bien distinguer les remblaiements alluviaux les uns par rapport aux autres et apportent de bons indices pour définir une chronologie relative des nappes. Des études comparatives ont également permis d'établir des corrélations avec les vallées affluentes de la Garonne (Icole et Rieucau 1982b ; Icole 1973 ; Icole 1980).

Depuis le milieu des années 80, les études menées dans le Midi toulousain sont beaucoup moins nombreuses. Ce court exposé montre combien l'apport de nouvelles données paraît indispensable à l'avancement des connaissances dans les vallées de la région qui nous intéresse. Les documents chronologiques fiables ont souvent fait défaut, laissant de nombreuses questions sans réponse ou bien sujettes à controverses. L'archéologie préventive, par son approche pluridisciplinaire, est à même de fournir des informations d'un intérêt certain.

\section{3 - Historique des recherches archéologiques}

13 Le renouveau des recherches sur les périodes anciennes du Paléolithique en Midi toulousain est essentiellement marqué par la communication de J. Jaubert C. Farizy et Ch. Servelle en 1989 au colloque de Saint-Riquier (Jaubert et Servelle 1996). Cette dernière présentait un état de la recherche sur l'Acheuléen dans le bassin de la Garonne au sens large, offrant ainsi une classification réactualisée de cette culture.

Parallèlement, des programmes de prospections systématiques de surface sont lancés, aboutissant à la découverte de nombreux sites inédits attribuables à l'Acheuléen (Millet 1994) ou au Moustérien (Pinos 1999). Ponctuellement, des sondages ont pu être effectués, ce qui a permis à leurs auteurs d'apporter de nouveaux éléments d'ordre pédo-stratigraphique (Millet D. et F. 1996). Cependant, les recherches prennent véritablement un nouvel essor à partir de 1997 avec la multiplication des opérations d'archéologie préventive sous l'impulsion conjointe du Service Régional de l'Archéologie de Midi-Pyrénées et de l'Association pour les Fouilles Archéologiques Nationales (AFAN). 
15 En effet, le développement économique croissant des communes limitrophes de la métropole régionale ainsi que l'exploitation régulière des molasses par les tuileries conduisaient à une réduction croissante des zones à fort potentiel en sites paléolithiques. Les interventions archéologiques d'abord ciblées sur des secteurs connus pour la richesse des vestiges repérés en coupe et/ou en surface se sont donc étendues par la suite à des aménagements impliquant des superficies importantes comme les lotissements. Ainsi, au cours de l'année 1997, deux opérations menées par l'AFAN venaient enrichir sensiblement notre compréhension de l'évolution des industries acheuléennes et des contextes sédimentologiques qui les caractérisaient. À En Jacca-La Sauvegarde (Colomiers, Haute-Garonne) l'étude typo-technologique d'une série de plus de trois cents pièces prélevée in situ associée à des analyses sédimentologiques, constituait un premier référentiel pour l'Acheuléen moyen du Midi toulousain (Capdeville et al. 1997).

Une autre évaluation située sur le talus de la moyenne terrasse à Fonsorbes (HauteGaronne), permit d'identifier une deuxième industrie aux caractères plus évolués (outillage lourd très discret, productions plus développées de supports moyens à petits) mais s'inscrivant dans un contexte sédimentologique très proche de celui décrit à $\mathrm{En}$ Jacca-La Sauvegarde (Capdeville et al. 1999).

17 Par ailleurs, une thèse sur le Paléolithique inférieur en Aquitaine méridionale, soutenue en 2001 par D. Millet, comportait une partie importante relative à la définition des « techno-complexes acheuléens » des terrasses de la Garonne en Midi toulousain (Millet 2001). Ce travail s'est développé à partir de l'étude de stations de plein air découvertes lors de prospections de surface. L'observation de coupes de carrières (En Jacca-Puntis à Colomiers), de sondages ponctuels effectués sur deux sites (Le Nan à Grenade-surGaronne et En Tole à Rieumes) et enfin la reprise des résultats d'une opération de sauvetage de l'AFAN à Colomiers (Capdeville et al. 1997), viennent compléter l'ensemble analysé. Bien que des éléments de réflexions aient été ainsi apportés sur la technologie lithique et les contextes "morpho-sédimentaires" considérés (op. cit. p. 518-536), il n'en reste pas moins que la perception chrono-culturelle de l'Acheuléen ne pouvait être véritablement renouvelée avec les données disponibles.

En 2002, était publié un article de synthèse rédigé par J. Jaubert qui récapitulait l'ensemble des informations et découvertes autorisant une présentation du Paléolithique dans le Midi toulousain (Jaubert 2002). Si l'attribution des vestiges (galets taillés ou façonnés) au Pré-Acheuléen découverts sur la nappe culminante (Vézian 1991) restait à confirmer, les industries acheuléennes, quant à elles, étaient mieux connues. L'Acheuléen moyen archaïque tel qu'il a été reconnu et défini dans le Tarn (Tavoso 1986) ne trouverait d'équivalent que dans les industries roulées signalées notamment à Mondavezan (Breuil 1937 ; Breuil et Méroc 1950). La phase moyenne ou classique, illustrée par des industries comprenant de l'outillage lourd (chopper, bifaces, hachereaux) et une production d'éclats non Levallois, s'avérait particulièrement bien représentée dans des gisements de surface, répartis sur une centaine de kilomètres en rive gauche de la Garonne. L'Acheuléen supérieur qui voit apparaître le débitage Levallois semblait identifié sur la seule station de La Baraque à Vacquiers (HauteGaronne) mais présentait déjà des indices de transition évidents vers le Paléolithique moyen (Jaubert 2002 p. 49). Ce dernier est également associé aux épandages alluviaux. D'importantes séries en silex récoltées à Cambernard (Haute-Garonne) ont fait l'objet 
d'une étude détaillée que l'ont peut considérer comme la première pour un gisement appartenant au Moustérien de Tradition Acheuléenne (Pinos 1999).

Cet état de la recherche mettait en évidence le déficit évident en données biochronostratigraphiques et radionumériques indispensables afin de disposer d'un cadre chrono-culturel plus fiable (Jaubert 2002 p. 46). Par ailleurs, l'élaboration d'un référentiel de gisements fouillés plus étoffé constitue un objectif tout aussi impératif dans la perspective d'une meilleure connaissance de l'évolution de l'Acheuléen et du Paléolithique moyen dans la diversité des contextes géomorphologiques du Midi toulousain.

\section{3 - Nouvelles données}

Depuis ces trois dernières années, l'AFAN puis l'INRAP, ont multiplié les diagnostics et les opérations de fouilles sur les terrasses du fleuve en Haute-Garonne. Le suivi des extensions des carrières (Briquetteries d'En Jacca à Colomiers), l'intervention sur certains projets de lotissements (Fonsorbes) et de ZAC, notamment celle d'Aéroconstellation à Blagnac, ont apporté de nouvelles données sur l'évolution des industries du Paléolithique ancien et moyen. Cependant, c'est au cours de la phase de diagnostic archéologique sur le fuseau à très grand gabarit de l'A 380 (ITGG A 380), que la présence d'un géomorphologue à plein temps (L. Bruxelles) et plus ponctuellement de micromorphologues (A.-L. Berthet et G. Delfour) a été déterminante. En effet, la confrontation constante entre les problématiques archéologiques et les observations géomorphologiques ou sédimentologiques, a abouti à la définition de trois types de contexte recelant des sites :

- le talus moyenne terrasse/basse terrasse ;

- les bords d'entailles internes de la moyenne terrasse ;

- l'intérieur des terrasses.

21 Cette collaboration, qui s'est poursuivie au cours des évaluations a largement contribué à une meilleure appréciation de certains sites et ainsi à préparer plus judicieusement les modalités de fouille. Les résultats de ces dernières récemment terminées ou encore en cours, sont exposés de manière succincte en l'attente de publications monographiques.

\subsection{Le talus moyenne terrasse/basse terrasse}

\section{Le site de Rominguière (Cornebarrieu)}


Figure 2 - Coupe géologique au droit du site de Rominguière (Cornebarrieu, Haute-Garonne) (dessin L. Bruxelles/INRAP).

Figure 2 - Geomorphological context of Rominguière site (Cornebarrieu, Haute-Garonne).

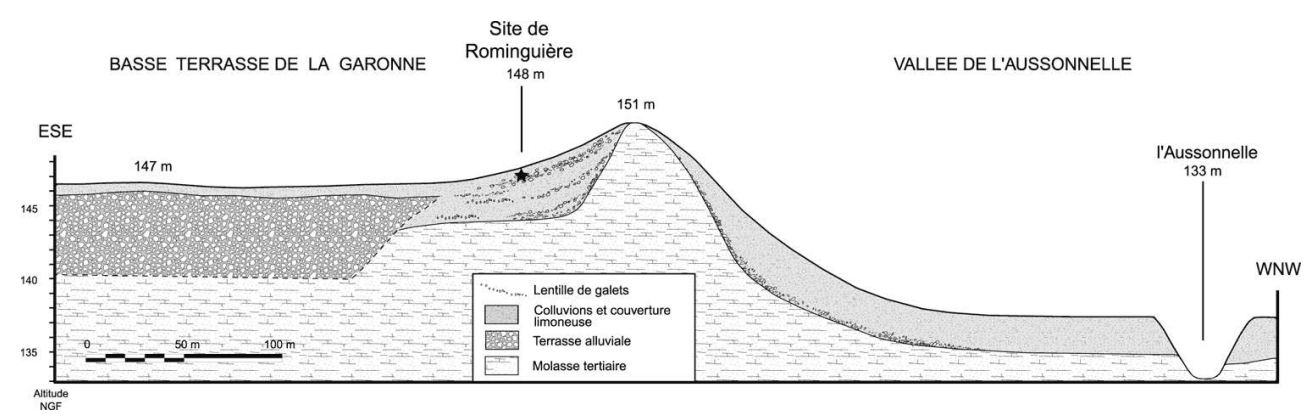

Le gisement de Rominguière a été détecté dans le cadre des opérations de diagnostic et de fouilles archéologiques préventives préalables à la construction de l'ITGG A380 (diagnostic P. Chalard dir.).

Les nombreuses pièces découvertes pendant l'évaluation (Colonge et al. 2003b) et la fouille (L.-A. Lelouvier dir., étude en cours) se localisent à la base d'un petit relief dominant la basse terrasse (fig. 2). A son sommet, la moyenne terrasse n'existe plus et seule la molasse tertiaire affleure et sous-tend une longue échine dissymétrique. Le versant ouest est relativement raide et domine la vallée de l'Aussonnelle d'une trentaine de mètres. En revanche, le versant est se raccorde avec moins de vigueur à la basse terrasse, une quinzaine de mètres en contrebas. Ce dernier est largement empâté par une accumulation de colluvions constituée de galets (dont certains de gros modules) qui atteint jusqu'à trois mètres d'épaisseur.

Il s'agit de colluvions fossiles, issues de l'érosion de la moyenne terrasse et de son soubassement molassique, avant le creusement de la vallée de l'Aussonnelle. La réalisation d'une coupe géologique à partir d'une trentaine de sondages permet même de penser qu'une partie de ces colluvions sont pénécontemporaines du remblaiement alluvial de la basse terrasse. Lors de la mise en place de cette accumulation colluviale, les alluvions de la moyenne terrasse recouvraient encore la molasse qui affleure au sommet de l'échine. Le décalage systématique des cours d'eau régionaux vers l'est a progressivement démantelé ce lambeau de moyenne terrasse dont il ne reste aujourd'hui que les colluvions.

25 La position du matériel archéologique est très instructive puisqu'il est inclus dans ces remaniements.

Ainsi, l'occupation originelle devait être localisée au sommet du talus, sur ce qui devait constituer un témoin plus ou moins large de la moyenne terrasse. Par la suite, le recul du talus par érosion a permis le piégeage du matériel au pied du versant est, tandis que le versant ouest de la butte témoin était sapé par le déplacement du lit de l'Aussonnelle. La vallée de ce petit cours d'eau était moins développée qu'à l'heure actuelle pendant l'occupation du site et de larges lambeaux de moyenne terrasse existaient encore sur sa rive droite.

Le gisement recèle une couche archéologique unique sur une épaisseur d'un mètre environ et elle est néanmoins particulièrement dense avec plusieurs milliers de pièces lithiques. 
La configuration du site témoigne clairement d'une position secondaire de l'industrie. La position du mobilier, concentré dans la partie concave du versant, juste avant l'amorce du replat, plaide en ce sens.

Les matières premières employées par les préhistoriques pour leur industrie sont en très large majorité des roches métamorphiques et sédimentaires de la chaîne axiale des Pyrénées (quartzites, schistes tachetés, lydiennes ...). Elles sont disponibles dans les alluvions de la Garonne sous forme de galets de différents modules selon les couches de graves affleurantes ou l'étage de terrasse concerné. Cet ensemble est complété par une pièce en silex, évoquant les silicifications des Petites Pyrénées. Ce matériau est connu dans les dépôts de la Garonne, aux alentours de Toulouse. L'approvisionnement en matières premières lithiques semble donc exclusivement local.

La phase de production est marquée par un faible développement des méthodes employées (principalement le débitage discoïde et sur enclume) et par une exploitation limitée des nucléus. La phase de transformation se distingue par un outillage retouché peu abondant et par une composante lourde plus fournie: bifaces, unifaces et hachereaux... (fig. 3). Certaines caractéristiques techno-typologiques propres à cette industrie présentent des aspects peut-être plus archaïques que les industries de l'Acheuléen moyen connues dans le Midi toulousain comme par exemple En Jacca-LaSauvegarde (Capdeville et al. 1997).

3 Ce site, caractérisé par une forte concentration de mobilier dans un espace bien délimité, pourra constituer une référence supplémentaire dans un corpus encore peu étoffé dans le contexte du bassin de la Garonne.

\section{Indice de site : rue Bellevue (Fonsorbes)}

2 L'opération de diagnostic « Rue Bellevue » conduite sur un projet de lotissement, s'est développée sur le bord de la moyenne terrasse de la Garonne ainsi que sur la pente correspondant à son talus de transition avec la basse terrasse. Cette intervention a permis de confirmer les observations géomorphologiques et sédimentologiques déjà faites dans des contextes similaires (talus de la moyenne terrasse) et de prélever une trentaine de quartzites taillés attribuables à des périodes anciennes du Paléolithique (Chalard et Onézime 2003).

33 Dans la partie sommitale de l'emprise, la nappe alluviale apparaissait sub-affleurante (nombreux galets en surface). Les sondages effectués dans la pente et au pied du talus ont démontré l'importance des phénomènes colluviaux. Les couches de graviers sont recouvertes par différents ensembles : niveaux remaniés (limons bruns à rares galets et fragments de briques) et limons jaune-orangé à traces d'hydromorphie. La variabilité du modelé du talus dans le secteur sondé est à l'origine de variations latérales et stratigraphiques des faciès sédimentologiques (érosions et colluvionnements différentiels).

Les quelques pièces débitées ont été découvertes in situ à l'interface d'un limon jauneorangé et d'une passée graveleuse. Pour mémoire, on rappellera qu'une évaluation menée en 1997 dans le même contexte à quelques centaines de mètres au nord du secteur de la « Rue Bellevue », débouchait sur la découverte d'une industrie considérée comme de l'Acheuléen supérieur (Capdeville et al. 1999). 
Figure 3 - Rominguière (Cornebarrieu, Haute-Garonne), industrie lithique en quartzite. 1 = uniface à pointe ; 2 = nucléus (dessins $\mathrm{D}$. Colonge/INRAP).

Figure 3 - Rominguière (Cornebarrieu, Haute-Garonne), quartzitic lithic industry. 1 = uniface, 2 = core.

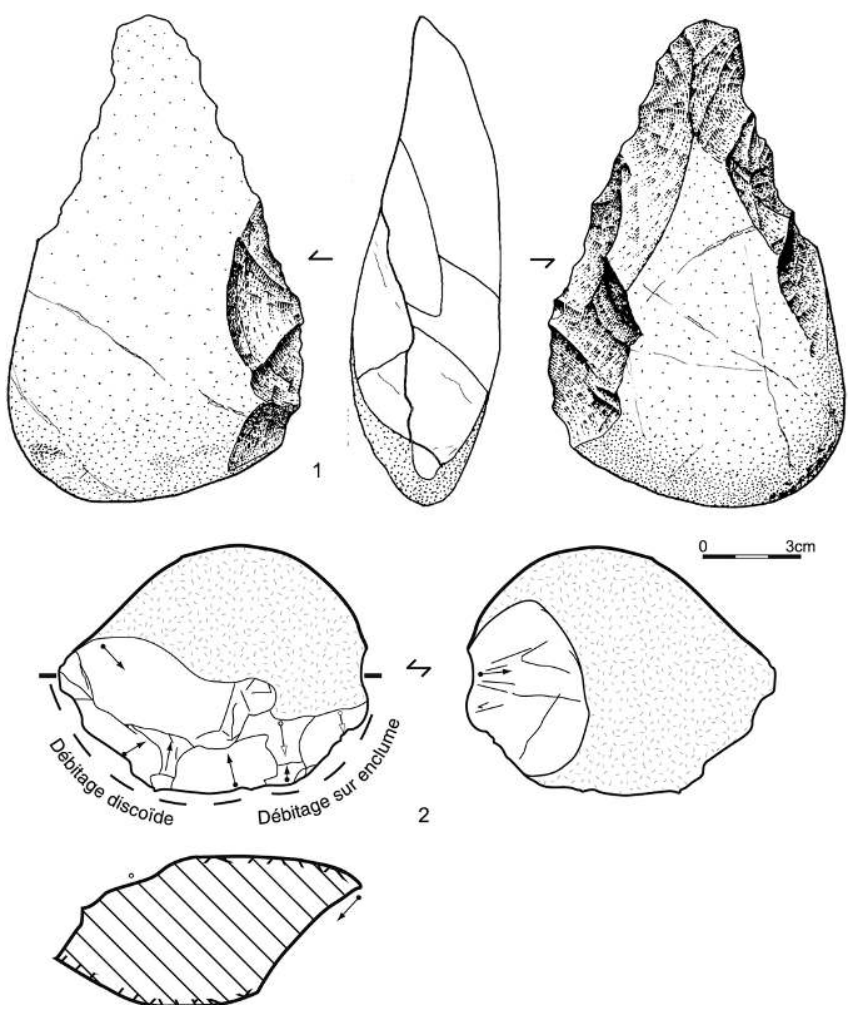

\section{2 - Les bords d'entailles internes de la moyenne terrasse}

\section{Le site de Bichou 2 (Montaigut-sur-Save)}

Evalué dans le cadre des travaux d'aménagement de l'ITGG A380 (évaluation L.-A. Lelouvier, étude en cours), ce site est localisé sur la moyenne terrasse de la Garonne. Celle-ci est ici en cours de dissection par une série de ruisseaux tributaires de la Save (fig. 1). Le ruisseau de la Croix s'est développé par érosion régressive dans la molasse sur plus de trois kilomètres. L'extrême amont actuel se situe au niveau des sites de Bichou et s'inscrit dans la moyenne terrasse.

Le matériel provient de l'érosion de la partie supérieure de la terrasse et les pièces sont préservées en position secondaire dans la formation colluviale. Plus précisément, elles se situent entre le point haut de la grave, où le site était semble-t-il localisé, et le talweg matérialisant le début du vallon de la Croix.

Lors de l'occupation du site, la vallée de la Save était déjà largement entaillée. Elle s'écoulait au minimum une trentaine de mètres en contrebas du toit de la moyenne terrasse. De fait, le réseau de ruisseaux affluents, tels que celui de la Croix, était déjà en partie constitué. Il est donc possible que la position morphologique originelle du site était sur la moyenne terrasse, non loin de l'amont du vallon rejoignant la vallée de la Save. Depuis, la poursuite du recul de la tête du vallon par érosion régressive a partiellement démantelé le gisement. 
de très bonne facture. L'outillage léger n'est pas représenté, mais il faut sans doute voir ici les effets du tri. Cette industrie, dans ses grandes lignes, serait comparable à celle du Lotissement du Château à Fonsorbes (Capdeville et al. 1999) ou encore à celle de Raspide 2 à Blagnac (cf. infra).

\section{Le site de Labadie (Mondonville)}

Figure 4 - Coupe géologique au droit du site de Labadie (Mondonville, Haute-Garonne) (dessin L. Bruxelles/INRAP).

Figure 4 - Geomorphological context of Labadie site (Mondonville, Haute-Garonne).

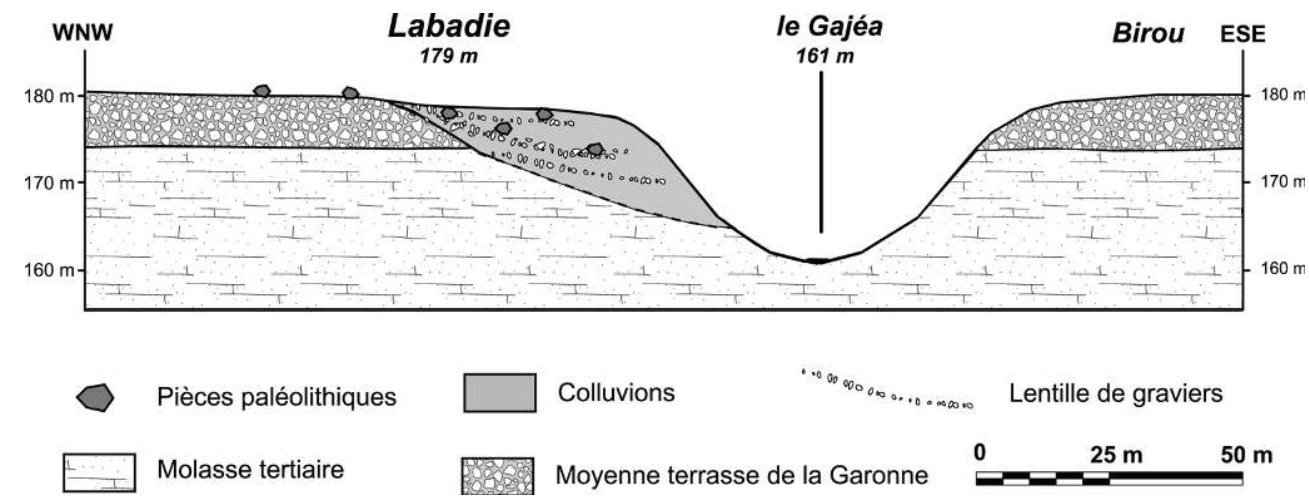

Le secteur de Labadie se trouve en bordure du vallon du Gajéa qui entaille la moyenne terrasse d'une vingtaine de mètres. Le site a été évalué dans le cadre des travaux d'aménagement de l'ITGG A380 (Arnoux et al. 2003). Il a fait l'objet d'une mesure conservatoire.

Les différents sondages ont révélé un contexte sédimentaire particulier (fig. 4). Le toit de la terrasse sub-affleurant à l'ouest plonge brutalement vers l'est sous une épaisse couverture de colluvions, non loin du versant de la vallée. Cette discordance matérialise l'érosion de la moyenne terrasse puis sa fossilisation par plusieurs mètres de colluvions.

Le corps de la moyenne terrasse est extrêmement altéré. Au sommet du corps alluvial, une dizaine de centimètres de petits graviers proviennent du remaniement de la partie supérieure de la grave. On ne retrouve plus dans cette formation que les roches les plus résistantes à l'altération telles que les quartz (le plus souvent éclatés), les quartzites, les lydiennes (Icole 1982b). Ce niveau de petits graviers qui surmonte directement la grave à l'ouest se prolonge en aval, au sein de la masse de colluvions, par plusieurs lits 
superposés. Ces niveaux, en forte pente au contact de l'ancien versant, passent progressivement à des lentilles discontinues et faiblement inclinées vers le talweg.

Le matériel archéologique est contenu le plus souvent dans des lentilles de graviers. On le retrouve donc sur toute l'épaisseur des colluvions, quasi systématiquement associés à ces petits niveaux détritiques. Il fait partie des colluvions et provient d'une occupation qui se localisait sur la moyenne terrasse.

L'examen des pièces confère à ce gisement de la vallée de la Garonne un caractère exceptionnel à plus d'un titre. Tout d'abord, la fraîcheur des pièces est inhabituelle et les faces taillées ne présentent quasiment pas de traces d'érosion ni d'éolisation. Ceci s'explique par le mode de conservation du matériel qui n'est pas resté emprisonné à la surface de la terrasse. Il a été piégé en contrebas et a rapidement été recouvert par d'autres colluvions. Par ailleurs, les remontages (fig. 5) réalisés entre des pièces provenant de différents niveaux de graviers démontrent l'origine commune de l'ensemble du matériel (cinq remontages et un appariement sur 175). Il provient d'un site unique dont les pièces ont été redistribuées dans plusieurs niveaux, au gré des ruissellements et des coulées boueuses qui affectaient le bord de la terrasse. 
Figure 5 - Labadie (Mondonville, Haute-Garonne). Figure 5 - Labadie (Mondonville, Haute-Garonne).

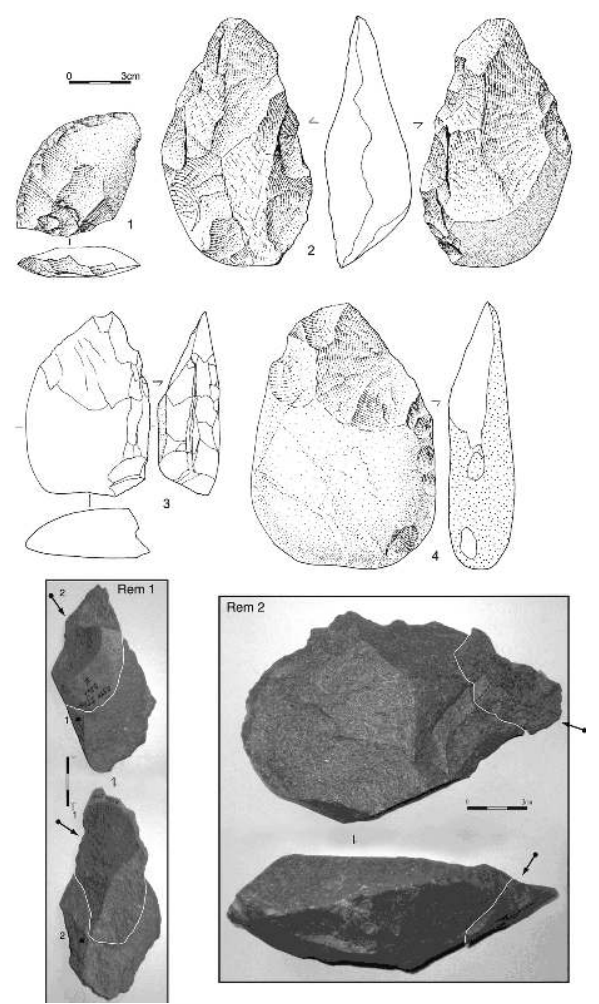

En haut : industrie lithique en quartzite :

1 = racloir convexe

2 = biface partiel à pointe

$3=$ « racloir » épais sur galet ; 4 = uniface partiel.

En bas : deux remontages (dessins et photos

M. Jarry/INRAP).

Up: lithic industry in quartzite. $7=$ convex scraper;

$2=$ partial biface;

$3=$ thick « scraper » on pebble;

$4=$ partial uniface.

Down: two reffitings.

Enfin, l'épaisse accumulation de colluvions et leur architecture traduisent le comblement complet du vallon du Gajéa. Ainsi, l'amortissement rapide de la pente soulignée par les niveaux de graviers et la dynamique plutôt faible dans ce contexte de colmatage n'ont pas permis l'exportation du matériel. Les pièces les plus lourdes, mais aussi les éclats sont donc restés sur place, fossilisés par la poursuite du comblement du vallon.

Les éléments de datation absolue de cette évolution géomorphologique (creusement du vallon, remblaiement puis recreusement des colluvions) font malheureusement défaut. Il est tentant de corréler cette histoire aux grands épisodes morpho-sédimentaires qui rythment l'évolution de la vallée de la Garonne sans pouvoir préciser si le colmatage du vallon du Gajéa correspond au remblaiement du dernier ou de l'avant-dernier épisode glaciaire. Néanmoins, l'état d'altération de la grave, qui paraît déjà très avancé lors de la mise en place des colluvions, tendrait à laisser penser qu'au moins un interglaciaire s'est écoulé avant le remaniement du matériel de la terrasse. 

éléments diagnostics suffisants pour permettre une caractérisation chrono-culturelle (fig. 5). L'échantillon de matières premières utilisé correspond au cortège pétrographique accessible au sein des alluvions de la Garonne, sans gestion particulière des matières premières. La phase de production est marquée par le faible développement des méthodes utilisées. Les produits récoltés sont cependant nombreux. Les outils sur éclat, contrairement à l'outillage lourd, restent peu abondants et mal caractérisés.

Tous ces éléments, avec notamment le faible développement des méthodes de débitage et la présence d'un outillage lourd bien caractérisé, permettent d'interpréter la série comme étant une manifestation de l'Acheuléen garonnais. Paléolithique moyen comme Raspide 2 à Blagnac (cf. infra), le Lotissement du Château à Fonsorbes (Capdeville et al. 1999), ou plus loin Esclignac à Montmaurin (Jarry 1994) ou les Bosses dans le Lot (Jarry et al. à paraître; Jarry et al. 2001a). Dans ces séries, les méthodes de débitages sont plus abouties, le débitage Levallois est parfois attesté, une gestion des matières premières est observable, l'outillage sur éclat se développe. Le niveau supérieur du site de Bichou 1 à Montaigut-sur-Save fait partie de ces gisements.

Par contre, les caractères de la série de Labadie offrent beaucoup plus de similitudes avec celles de la phase dite «classique » de l'Acheuléen. Les industries d'En Jacca - La Sauvegarde (Capdeville et al. 1997) ou d'En Jaca (Méroc 1961) à Colomiers, plus loin de Campsas (Tavoso 1986) ou encore Rieumes à En Tole (Millet D. et F.1996), pour n'en retenir que quelques unes, sont les quelques références comparables. Bien sûr, dans certaines de ces collections, issues généralement de ramassages de surface, les « coupsde-poing " sont souvent présents dans des proportions exagérées.

Le site acheuléen de Rominguière à Cornebarrieu (cf. supra), est sans doute assez proche de cette série. Ces deux gisements seront sans nul doute complémentaires.

\section{3 - L'intérieur des terrasses}

\section{Les sites d'En Jacca (Colomiers)}

Le secteur d'En Jacca (fig. 6) à trois kilomètres à l'ouest de Colomiers, est connu depuis de nombreuses années pour les séries acheuléennes qui y sont découvertes. Dès 1959, les exploitations de la molasse pour les briqueteries permettaient à L. Méroc d'identifier en stratigraphie des industries attribuées à l'Acheuléen reposant sur le toit de la grave de la moyenne terrasse (Méroc 1961). Depuis, des prospections assidues et des opérations d'archéologie préventive n'ont cessé dans ce périmètre. 
Figure 6 - Localisation des opérations archéologiques sur la zone d'En Jacca (dessin M. Jarry/ INRAP).

Figure 6 - Location of archaeological projects in the En Jacca area.

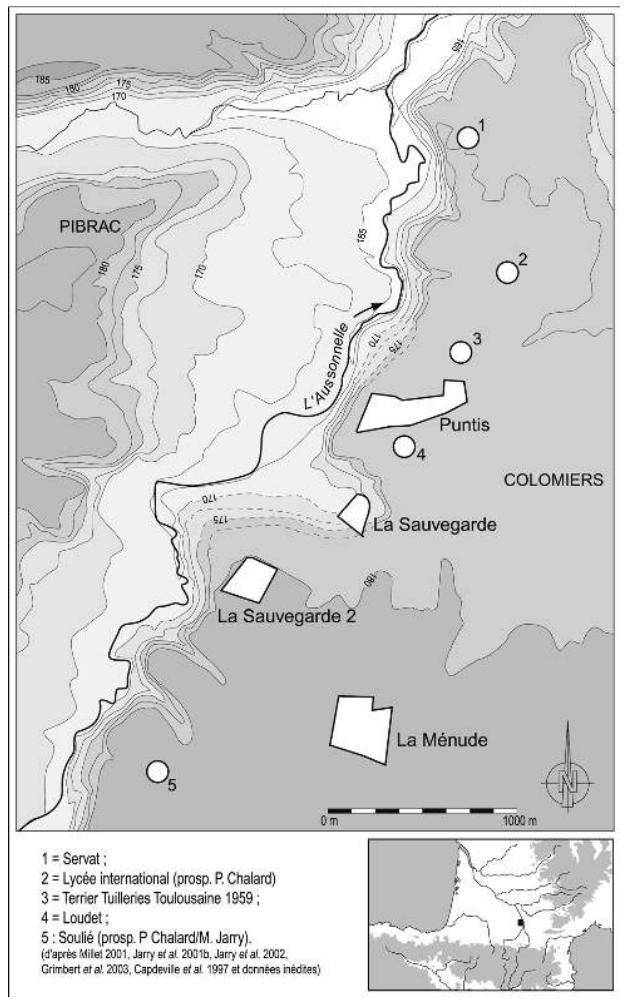


Figure 7 - En Jacca (Colomiers, Haute-Garonne) quartzites taillés. Figure 7 - En Jacca (Colomiers, Haute-Garonne) lithic industry in quartzite.

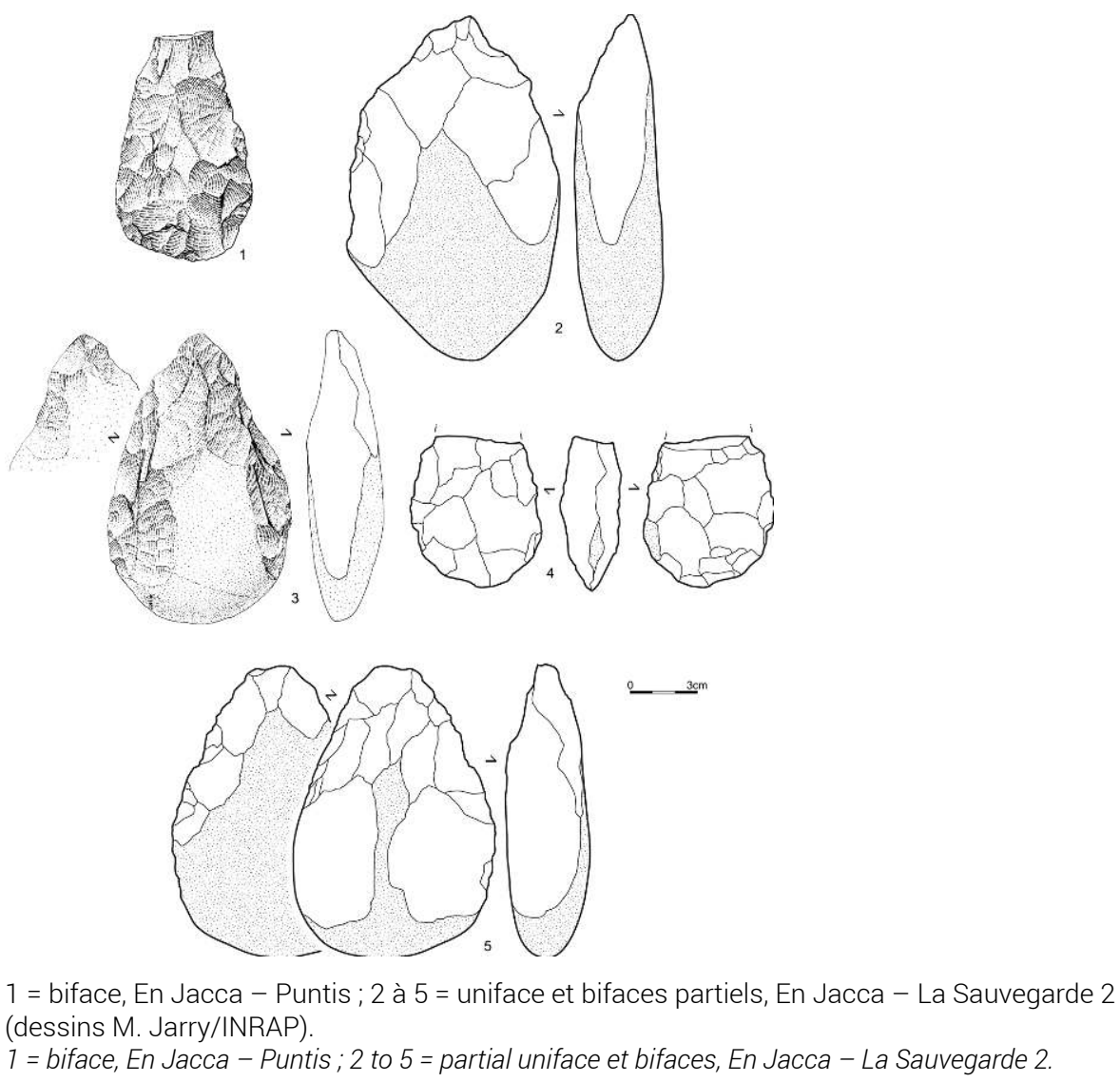

En 2001, une extension de carrière permettait de reconnaître une vaste zone au sein de la moyenne terrasse : En Jacca - Puntis (Jarry et al. 2001b). À environ 100 mètres à l'ouest et à une trentaine de mètres en contrebas, la terrasse est recoupée par le réseau hydrographique de l'Aussonnelle. Les trois coupes relevées sur le front de carrière montrent une stratigraphie très variable. Le sommet du corps alluvial est subaffleurant dans certains secteurs. On retrouve alors de l'industrie lithique dans le niveau de labours. Mais la majorité du matériel provient des zones où les dépressions $\mathrm{du}$ corps alluvial ont permis une meilleure préservation des limons de couverture. Ces derniers peuvent alors atteindre près de deux mètres de puissance. Certaines pièces lithiques se retrouvent intégrées dans le sommet du corps alluvial de la terrasse, sur une dizaine de centimètres d'épaisseur. Cette position stratigraphique pourrait être liée à des phénomènes pédologiques ou, plus sûrement à des remaniements de la partie supérieure de la terrasse.

Un premier lot de vestiges lithiques, à l'éolisation bien marquée, renvoie de manière assez claire à l'Acheuléen «classique » de la région pyrénéo-garonnaise (fig. $7 \mathrm{n}^{\circ} 1$ ). Le second ensemble, plus modeste évoque très nettement le Paléolithique moyen. À titre d'exemple, deux nucléus s'apparentent étroitement à ceux de la série de Mauran (Jaubert 1993).

Une première tranche d'extension de la carrière d'En Jacca - la Sauvegarde avait livré une riche documentation attribuable à l'Acheuléen «classique » (Capdeville et al. 1997). Une deuxième tranche d'extension a permis en 2002 d'effectuer des reconnaissances 
sur une zone plus en retrait du bord de taille de l'Aussonnelle (Jarry et al. 2002). En effet, le site de d'En Jacca - La Sauvegarde 2 est situé au cœur de la moyenne terrasse. Il surplombe d'une vingtaine de mètres la rivière. Comme pour En Jacca - La Sauvergarde, la couverture limoneuse a été affectée par des remaniements d'origine anthropique et présente donc une épaisseur variable comprise entre quelques centimètres et un mètre. L'industrie lithique provient généralement des zones où les limons sont les mieux préservés.

Mais les relations entre la concentration des vestiges et les variations topographiques du corps alluvial semblent moins sensibles que sur les autres sites (En Jacca - La Sauvegarde et Puntis). La petite série récoltée correspondrait plus à des vestiges épars qu'à un véritable site. Elle se répartit sans véritable concentration dans toutes les parcelles reconnues. Cependant, l'ensemble n'apparaît pas aberrant (fig. 7, $n^{\circ} 2$ à 5) et s'intègre sans difficultés dans les séries acheuléennes garonnaises, et notamment En Jacca - La Sauvegarde.

59 Un peu plus loin, à En Jacca - La Ménude, un autre diagnostic a livré quelques pièces lithiques isolées (éclats, choppers, nucléus...) (Grimbert et al. 2003). Cette documentation, très ténue et apparemment répartie de manière aléatoire, témoigne au cœur de la terrasse de zones fréquentées mais exemptes de gisements.

\section{ZAC Aéroconstellation (Blagnac) : découvertes ponctuelles et site de Raspide 2}

L'ensemble des opérations menées sur la ZAC Aéroconstellation à Blagnac nous a offert, pour la première fois, une perception large de l'occupation paléolithique de ce secteur de la basse terrasse de la Garonne. Sur les 150 hectares diagnostiqués (Pons 2001) un certain nombre d'indices a pu être relevé et complété à l'occasion des fenêtres de fouilles des sites néolithiques et protohistoriques.

61 Tout d'abord, un biface et un nucléus, roulés, découverts dans la grave sur le site protohistorique de Raspide 1 (C. Sireix dir.) attestent que des artisans acheuléens fréquentaient la vallée de la Garonne pendant l'avant dernier cycle alluvial. Les autres indices, présents sous la forme de ce qui apparaît comme un épandage discontinu mais homogène, semblent le reflet d'une fréquentation dense de la vallée par des groupes préhistoriques porteurs d'une culture matérielle Acheuléen supérieur en cours d'évolution vers le Paléolithique moyen.

Un site, Raspide 2, a fait l'objet d'une évaluation (Jarry et Colonge 2001) et d'une fouille (Colonge et al. 2002). Il est localisé au centre de la basse terrasse de la Garonne. La stratigraphie est relativement homogène. On observe des limons d'environ $70 \mathrm{~cm}$ d'épaisseur, recouvrant la grave dont seule la partie superficielle a été mise au jour. Dans l'angle nord-est du gisement, une dépression du toit de la nappe alluviale, correspondant probablement à un paléo-chenal, a permis l'accumulation de prés d'1,50 $m$ de limons.

Différentes analyses (granulométrie, morphoscopie et micromorphologie) ont été menées afin de comprendre l'origine et la mise en place de la couverture limoneuse à la base de laquelle se situent les vestiges archéologiques. En effet, ces dépôts peuvent trouver leurs origines potentielles dans les couvertures limoneuses de la moyenne terrasse et/ou dans la molasse, leur nature et leur granulométrie étant comparables. Des matériaux éoliens, sous la forme de grains de quartz esquillés, remaniés ou bien 
contemporains de la formation des limons, ont aussi été identifiés à l'échelle microscopique.

Concernant la dynamique sédimentaire, il apparaît que la couverture limoneuse actuelle sur laquelle s'est développé un sol lessivé hydromorphe, est une colluvion de sol. Plusieurs indices microscopiques (nodules ferro-manganiques, papules) témoignent de la reprise d'un ou plusieurs anciens horizons pédologiques. D'après la présence de galets à la base des limons, il est probable que ces perturbations avaient aussi affecté le sommet de la grave. Le matériel archéologique fait donc partie intégrante des matériaux colluviés.

La série lithique (fig. 8) issue de cette fouille (près de 1000 pièces) renouvelle sensiblement les cadres traditionnels du Paléolithique ancien et moyen du parcours toulousain de la vallée de la Garonne. En premier lieu, elle porte les stigmates bien marqués d'une usure éolienne. Or, une chronologie relative avait été établie sur la base des états physiques et de la position des séries au sein des étages de terrasse. L'éolisation prononcée des artefacts apparaissait comme le marqueur des séries acheuléennes de la moyenne terrasse. Cette usure mécanique était alors classiquement considérées comme "rissienne ». Des industries de l'Acheuléen final, éolisées, sont connues dans la vallée de l'Agout (Tavoso 1986). Les aspects de surface étaient alors considérées comme attribuables au « Riss récent ». Ce critère n'est plus recevable et ce phénomène doit plutôt être corrélé aux phases de déflations (dépôts lœssiques).

Figure 8 - Raspide 2 (Blagnac, Haute-Garonne) industrie lithique en quartzite.

Figure 8 - Raspide 2 (Blagnac, Haute-Garonne) lithic industry in quartzite.

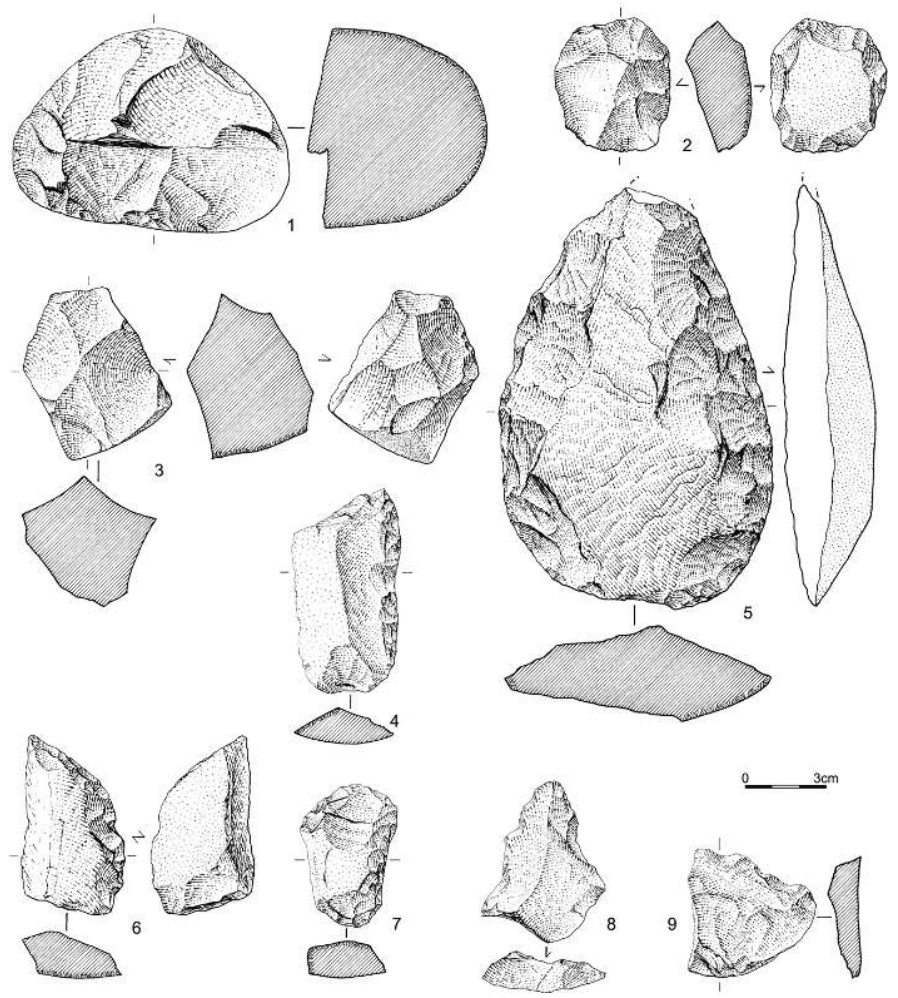

1 = nucléus sur enclume ; 2 = nucléus Levallois ; 3 = nucléus discoïde bifacial ; 4, 6 à 9 = outillage léger ; 5 = uniface à pointe (dessin M. Jarry/INRAP).

$1=$ core on anvil ; 2 = Levallois core ; $3=$ bifacial discoïd core ; 4,6 to $9=$ light tools ; 5 = uniface 
De manière synthétique, le gisement de Raspide 2 peut être rattaché aux ensembles de
transition entre le Paléolithique inférieur et le Paléolithique moyen, daté aux Bosses du
stade isotopique 9 .

De manière synthétique, le gisement de Raspide 2 peut être rattaché aux ensembles de
transition entre le Paléolithique inférieur et le Paléolithique moyen, daté aux Bosses du
stade isotopique 9 .

\section{Le site de Bichou 1 (Montaigut-sur-Save)}

L'acquisition des matières premières, en quasi-totalité des roches pyrénéennes métamorphiques ou sédimentaires de la chaîne axiale, complétées, comme à Rominguière, par deux éléments en silex des Petites Pyrénées, a été faite sur place, sous forme de galets au sein des formations alluviales. Les artisans préhistoriques ont légèrement privilégié les quartzites bleutés dans leur collecte mais, globalement, le panel de matière première exploité est très proche de la composition lithologique exploitable de la grave.

très affirmés, notamment pour la production. Cette dernière associe à la méthode Levallois un débitage discoïde développé et diversifié. L'outillage retouché à forte composante en racloirs avec des outils de type Paléolithique supérieur appuierait cette attribution chronoculturelle. Cependant, des traits anciens sont perceptibles. Les bifaces et hachereaux, bien que peu nombreux en sont la marque la plus symptomatique. La diversité des supports, la faible standardisation de l'outillage léger ainsi que l'absence d'économie des matières premières participent à cette impression.

L'occupation préhistorique est postérieure à l'édification de la basse terrasse. Elle s'inscrit sans difficulté dans la forme régionale "classique " de l'Acheuléen supérieur avec les gisements, entre autres, de La Baraque et d'En Régis dans le Tarn (Tavoso 1986), des couches intermédiaires et supérieures de la grotte de la Terrasse (Serra 1980) ou de la base de la grotte de Coupe-Gorge (Gaillard 1982) à Montmaurin (HauteGaronne). Les affinités sont aussi très fortes avec les sites d'Esclignac à Montmaurin (Jarry 1994) et des Bosses à Lamagdelaine dans le Lot (Jarry et al. 2001a et à paraître) tous deux attribués à une phase ancienne du Paléolithique moyen.

Proche de Bichou 2 (fig. 9) ce site a été évalué dans le cadre de la construction de l'ITGG A380 (Colonge et al. 2003a) et il est toujours en cours d'étude (M. Jarry dir.). 
Figure 9 - En haut : localisation des sites de Bichou 1 et 2 (Montaigut-sur-Save, Haute-Garonne). En bas : coupe schématique et log du site de Bichou 1 (dessin T. Arnoux/INRAP).

Figure 9 - Up : location of Bichou 1 et 2 sites (Montaigut-sur-Save, Haute-Garonne). Down : diagrammatic section and stratigraphic section of Bichou 1 site.
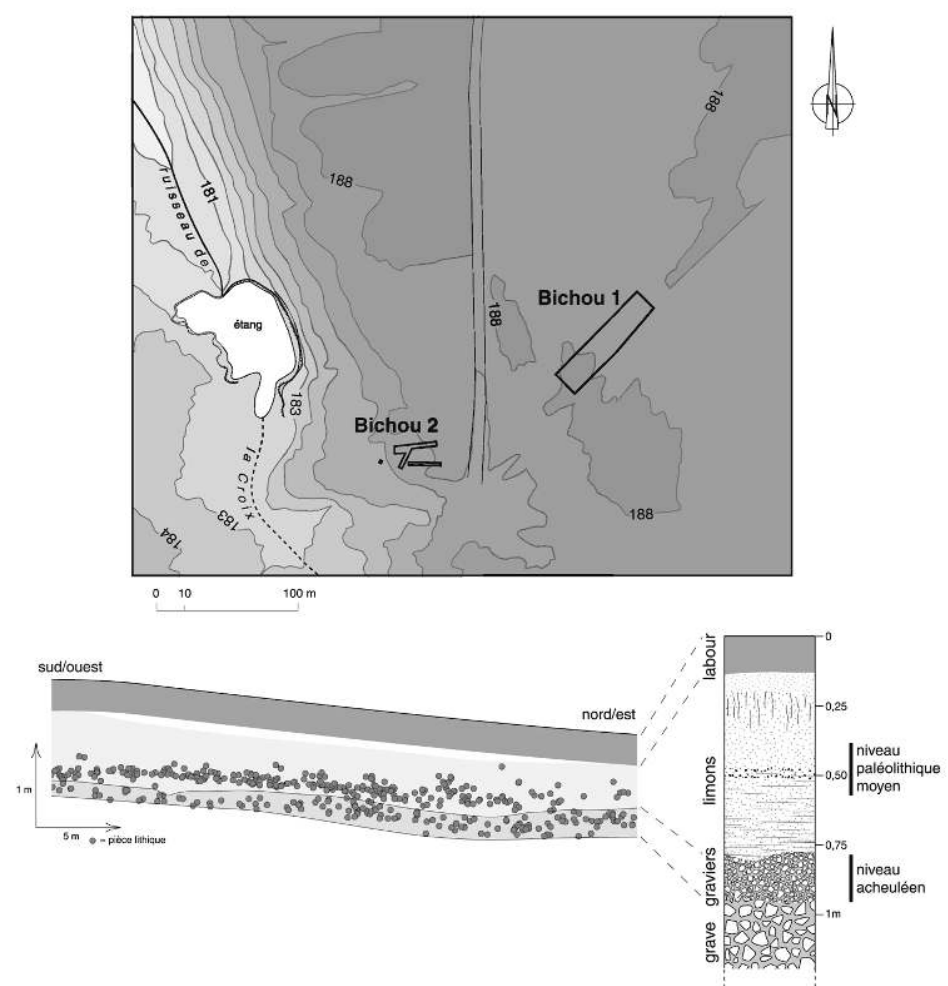

71 En fonction de sa position topographique, le matériel archéologique n'a pas été conservé dans les mêmes conditions. Alors que l'entaille du ruisseau de la Croix a érodé la partie occidentale (Bichou 2), la partie orientale (Bichou 1) est restée préservée sur la terrasse. Dans ce secteur, la disposition du matériel archéologique au sein de deux niveaux montre à la fois les dynamiques sédimentaires qui régissent la mise en place de la couverture limoneuse mais permet aussi d'appréhender, pour l'instant de manière relative, la durée du phénomène.

Le toit du corps graveleux d'une terrasse est assez irrégulier. Le type de sédimentation en chenaux ainsi que l'érosion postérieure de cette formation alluviale (crues, ruissellements) déterminent la coexistence d'une topographie très différente de l'aspect tabulaire que l'on connaît actuellement. C'est la constitution d'une couverture limoneuse (lœss, partie supérieure de la nappe alluviale remaniée, colluvions de sols) suivie par plusieurs siècles d'agriculture qui a contribué à niveler l'essentiel de ces formes. Ainsi, nous avons pu observer pendant la phase de diagnostic archéologique, cette variabilité. Le toit sub-affleurant dans certains secteurs passe progressivement à un, deux voire plusieurs mètres de profondeur.

73 Le site de Bichou 1 illustre bien ces variations : le toit de la grave qui est sub-affleurant à l'ouest s'abaisse progressivement vers l'est pour atteindre 1,30 mètres de profondeur. Le matériel archéologique est réparti en fonction de cette topographie puisque l'essentiel des pièces a été découvert dans la zone déprimée. Elles ont été remaniées et déplacées vers les dépressions.

L'analyse sédimentaire et minéralogique confirme ce remaniement et permet d'inscrire ce phénomène dans la durée. Les pièces les plus basses (Acheuléen) ont été trouvées 
dans un niveau à graviers, en continuité avec le corps alluvial à galets de la moyenne terrasse. Cependant, ces deux séquences présentent, comme nous l'avons observé sur le site de Labadie, des caractéristiques très différentes. La grave, marquée par une très forte altération est constituée de galets parfois tendres, dont certains de gros modules, pris dans une matrice argileuse. Immédiatement au-dessus, le niveau à graviers est composé d'éléments plus petits (jusqu'à $3 \mathrm{~cm}$ de diamètre), souvent anguleux, où n'apparaissent pratiquement plus que des quartz et des quartzites. Ce tri sédimentaire et minéralogique matérialise l'érosion et le remaniement de la grave altérée. Les points hauts de la grave ont été érodés et le matériel résiduel s'est accumulé dans les dépressions. À cette occasion, tous les éléments peu résistants ont été évacués sous forme de sable et d'argile tandis que les quartz et les quartzites, souvent fractionnés par l'altération, ont été remaniés. Ce niveau matérialise donc une remobilisation des éléments de la grave après une longue période d'altération. Ainsi, la mise en place de la couverture limoneuse est largement postérieure à la construction de la terrasse qui la supporte. L'intensité de l'altération, considérée comme progressive dans le temps (Icole et Rieucau 1982b), laisse penser qu'une très longue période sépare ces deux séquences sédimentaires. Au moins un interglaciaire, contexte le plus favorable à l'altération, est certainement compris dans cette discordance, entre la fin de la constitution de la moyenne terrasse et la mise en place du niveau à graviers.

Au-dessus du lit de graviers, le deuxième niveau contenant du matériel archéologique du Paléolithique moyen se situe au sein de la couverture limoneuse. Cette industrie, plus récente, témoigne de la mise en place puis de l'érosion d'un autre site archéologique pendant l'accumulation de limons au toit de la terrasse. Cette stratigraphie originale, encore en cours d'analyse, fournit une première approche de la durée de la constitution de la couverture limoneuse et révèle clairement une mise en place polygénique.

Les matières premières exploitées par les premiers occupants du site (niveau inférieur) (fig. 10, $n^{\circ} 6$ à 8) et leurs proportions sont celles classiquement observées dans les séries de l'Acheuléen moyen comme à Rominguière ou à Labadie. De la même façon, la production met en œuvre un débitage discoïde peu développé (modalité unifaciale partielle très majoritaire). Le débitage sur enclume est aussi présent. L'outillage retouché est rare, au profit d'une importante série d'unifaces, de bifaces et de hachereaux. Nous pouvons attribuer sans grand risque d'erreur cette série à la phase moyenne dite "classique» de l'Acheuléen garonnais (En Jacca, Rieumes, Labadie, Rominguière...). 
Figure 10 - Bichou 1 (Montaigut-sur-Save, Haute-Garonne). Figure 10 - Bichou 1 (Montaigut-sur-Save, Haute-Garonne).

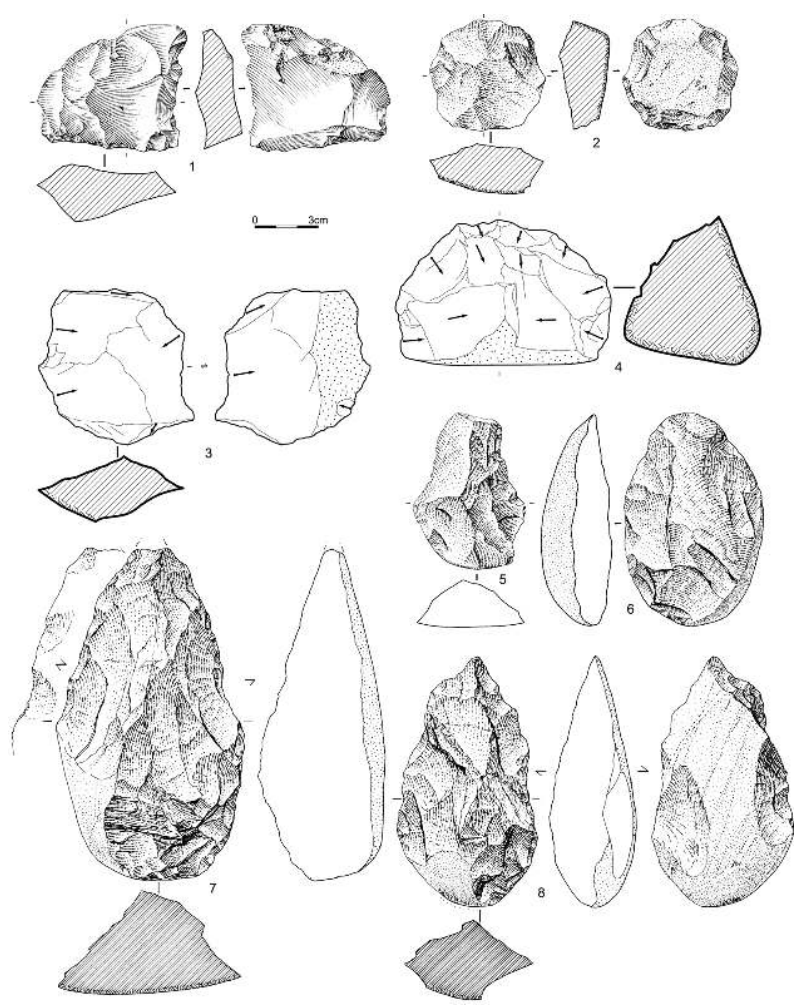

Niveau supérieur : 1 = nucléus Levallois, silex; 2 = nucléus Levallois, quartzite ; 3 = nucléus discoïde bifacial partiel ; 4 = nucléus discoïde unifacial partiel, quartzite ; 5 = éclat retouché, quartzite. Niveau inférieur : 6 et 7 = unifaces, quartzite ; 8 = biface partiel, quartzite (dessins M. Jarry/INRAP). Upper level : 1 = Levallois core, flint; 2 = Levallois core, quartzite; 3 = partial bifacial discoïd core ; 4 = partial unifacial discoïd core, quartzite ; 5 = retouched flake, quartzite. Lower level : 6 and 7 = unifaces, quartzite ; 8 = partial biface, quartzite.

77 Les caractéristiques de la série du niveau supérieur (fig. 10, $\mathrm{n}^{\circ} 1$ à 5) sont quant à elles assez différentes. Le débitage Levallois est attesté et bien développé, le débitage discoïde est complexe. La production est résolument orientée vers le débitage d'éclats de dimensions petites à moyennes. L'outillage léger est dominé par les encoches et les denticulés aux côtés de racloirs. La composante lourde n'est représentée que par des galets aménagés. Bifaces et hachereaux sont absents. Une sélection et la gestion affirmées des matières premières accessibles au sein des graves, comprenant notamment du silex, participent à l'attribution de cette série au Paléolithique moyen. Ce dernier reste indifférencié dans le cadre de cette présentation préliminaire.

Le caractère exceptionnel du site réside donc dans la conservation de deux niveaux archéologiques bien différenciés d'un point de vue stratigraphique. Séparés par une couche stérile, ils permettent enfin de dissocier deux séries qui, en d'autres lieux, auraient peut-être été réunies en une seule collection.

\section{4 - Synthèse}

L'exposé des différents contextes géomorphologiques retenus comme les plus caractéristiques mais aussi et surtout comme les plus prometteurs en terme de potentialités archéologiques, autorisent quelques réflexions sur la conservation et 
l'implantation des gisements. Par ailleurs, les premiers résultats issus majoritairement des évaluations et des études en cours de certains sites viennent alimenter le débat sur l'apport de l'archéologie préventive pour une structuration plus précise de l'Acheuléen et du Paléolithique moyen.

\section{Contextes favorables pour la conservation des sites}

(1) de réaliser des transects objectifs où les recherches archéologiques n'ont pas été influencées par le contexte topographique ou par la présence de sites connus. Pourtant, la répartition géographique des sites ne paraît pas aléatoire (cf. fig. 1). En effet, alors que les diagnostics concernent tous les contextes géomorphologiques de ce secteur de la vallée de la Garonne, seuls quelques-uns ont été régulièrement occupés. On peut dès lors avancer l'hypothèse qu'il s'agit d'un choix délibéré au profit de certaines morphologies. Ainsi, les grandes étendues planes de la moyenne terrasse semblent avoir été délaissées et n'ont livré que très peu d'indices majeurs. En revanche, les bordures qu'elles soient dues à l'incision de petits cours d'eau affluents (Bichou 2, 
Labadie) ou qu'elles dominent un autre niveau de terrasse (Rominguières) ont régulièrement livré des sites paléolithiques d'importance.

\section{L'apport de l'archéologie préventive pour la constitution de référentiels techno-typologiques}

86 À l'issue de cet exposé des séries lithiques découvertes dans le cadre d'opérations d'archéologie préventive récentes, il est aisé d'apprécier le potentiel de connaissances qui peut en être retiré. Si les récoltes de surface ont permis de dessiner les grands traits des cultures matérielles des hommes ayant parcouru le bassin garonnais, il n'en demeure par moins que la recherche semblait dans une impasse méthodologique. Il devenait impératif de renouveler notre vision de ce qui pouvait apparaître comme d'immenses « épandages » hétérogènes. Les premiers résultats concluants concernent l'approche territoriale: l'occasion de sonder systématiquement de vastes entités géomorphologiques a permis de repérer des «sites" au sein de ces innombrables témoins d'occupations aux périodes anciennes de la Préhistoire. Les variations latérales d'altitudes du toit de la grave et les entailles constituant autant de pièges pour les vestiges, la mise au jour de séries « complètes » est devenue possible et autorise enfin des études techno-typologiques sur des ensembles étoffés. Si l'homogénéité de ces séries peut toujours être discutée, les industries présentées ici apportent une réponse optimiste. Ainsi, le site de Labadie, avec ses remontages, offre pour la première fois dans la vallée de la Garonne l'assurance d'être en présence d'un ensemble cohérent. À cette amélioration qualitative du matériel d'étude dont les retombées sont facilement appréciables pour les analyses techno-typologiques, vient s'ajouter, avec le site de Bichou 1, le premier site stratifié de plein air de la région. Nous avons enfin, entre deux séries, une indication chronologique, même si elle reste très relative. Ce site permet par ailleurs de remettre en cause, au même titre que ce que nous avons vu pour Raspide 2, la pertinence du tri des séries d'après leur seule patine.

Fort de ces observations, il devient beaucoup plus raisonnable d'effectuer des études sur ce matériel. Les premières avancées qui peuvent être mises en avant ici concernent la précision ou la révision du cadre chrono-culturel, notamment pour les faciès de transition Paléolithique ancien / Paléolithique moyen. En effet, notre perception de la gestion - voire d'une économie - des matières premières de plus en plus affirmée, de la diversification du débitage discoïde et de l'apparition de la méthode Levallois dès les faciès de transition, se base sur les premiers résultats obtenus au terme de ces analyses. Les redéfinitions des complexes culturels ne peuvent que bénéficier de ces études, dans un contexte où les matières premières induisent un outillage retouché peu abondant et peu différencié.

\section{5 - Conclusion}

Cette présentation synthétique des dernières opérations en archéologie préventive dans le Midi toulousain constitue une première étape puisque l'ensemble des données, notamment celles relatives aux aspects typo-technologiques, n'a pas été entièrement dépouillé. Toutefois, les études géomorphologiques développées ont d'ores et déjà atteint un premier objectif puisque différents contextes sont maintenant bien individualisés. Le potentiel avéré ou envisagé pour chacun d'entre eux, participe ainsi à 
une meilleure préparation des interventions. Les propositions avancées quant aux modalités de conservation des gisements et à leur répartition ainsi que la nécessité de mettre au jour des industries homogènes et représentatives, devraient également contribuer à une programmation ambitieuse.

La caractérisation des industries acheuléennes et moustériennes et de leur faciès de transition exige d'intensifier la fouille de sites dans les contextes géomorphologiques favorables que nous avons identifiés. De plus, la création d'un référentiel de gisements impose de prendre en compte toutes les opportunités d'analyses susceptibles d'être effectuées. On prendra comme exemple l'intérêt qu'il faut porter aux séries comportant des remontages. En effet, ces derniers nous renseignent, entre autres, sur les pratiques techniques de l'individu, tout aussi utiles à appréhender que les méthodes de débitage mises en œuvre par les groupes culturels, par ailleurs mieux connues. Toutefois, un cadre chronologique élaboré sur la base de datations numériques fait encore défaut. L'existence de quelques pièces brûlées dans certains sites et les progrès de certaines méthodes de datations (OSL) laissent espérer à court terme des avancées significatives dans ce domaine. De même, la datation récente de malacofaune découverte dans la basse plaine et dans les limons de la basse terrasse de la Garonne apporte les premiers repères chronologiques (Tardiglaciaire) dans la mise en place des couvertures limoneuses des terrasses.

Ainsi, il n'est pas inutile d'insister de nouveau sur l'écart très important existant entre des bassins hydrographiques bien mieux documentés qu'ils fassent partie de l'Aquitaine (Dordogne, opérations de l'A 89 dans la vallée de l'Isle: Geneste et Gé 2001) ou plus lointains comme la vallée de la Somme (Antoine et al. 2003) et le Midi toulousain, encore déficitaire de ce point de vue. À l'évidence, l'archéologie préventive est amenée à jouer un rôle prépondérant dans la recherche sur le Paléolithique ancien et moyen du couloir garonnais, axe majeur pour ces périodes dans le Bassin Aquitain, trait d'union entre le monde pyrénéen aux forts accents ibériques (Colonge 2001 et à paraître) et les bordures du Massif central aux faciès particuliers (Jarry et al. 2001a et à paraître). Ce programme ambitieux ne pourra réellement prendre tout son essor qu'au sein de projets pluridisciplinaires et inter-institutionnels.

\section{BIBLIOGRAPHIE}

ANTOINE P., AUGUSTE P., BAHAIN J.-J., COUDRET P., DEPAEPE P., FAGNART J.-P., FALGUERES C., FONTUGNE M., FRECHEN M., HATTE C., LAMOTTE A., LAURENT M., LIMONDIN-LOZOUET N., LOCHT J.-L., MERCIER N., MOIGNE A-M., MUNAUT A.-V., PONEL P. et ROUSSEAU D.-D. 2003 Paléoenvironnements pléistocènes et peuplements paléolithiques dans le bassin de la Somme (nord de la France). Bulletin de la Préhistoire Française, t. 100, nº 1, pp. 5-28.

ARNOUX T., BRUXELLES L., CHALARD P., COLONGE D., JARRY M., ONÉZIME O. et coll. de BEVILACQUA-LEBAR R. et BATS J.-C. 2003 - Labadie (Mondonville, Haute-Garonne). Rapport d'évaluation archéologique, INRAP, Toulouse, $41 \mathrm{p}$. 
BOURGEAT, F., ICOLE et M., REVEL, J.-C. 1984 - Les terrasses alluviales dans les petites Pyrénées et l'avant pays molassique : les conditions de leur mise en place. In: Signification dynamique et climatique des formations et terrasses fluviatiles quaternaires. Bulletin de l'Association Française pour l'Etude du Quaternaire, nº 17-18-19, Colloque de l'Association Française pour l'Etude du Quaternaire, Paris. pp. 60-66.

BREUIL H. 1937 - Terrasses et quartzites taillés de la haute vallée de la Garonne. Bulletin de la Préhistoire Française, t. 34, n² 2, pp. 104-130.

BREUIL H. et MÉROC L. 1950 - Les terrasses de la Garonne et leurs quartzites. In : Préhistoire, Paris : PUF, pp 1-15.

CAPDEVILLE J.-P., CHALARD P., COLONGE D. et JARRY M. 1999 - La station acheuléenne du Lotissement du Château à Fonsorbes (Haute-Garonne). Bulletin Préhistoire du Sud-Ouest, n 6/1999/2. 1999, pp. 105-124.

CAPDEVILLE, J.-P., CHALARD, P., JARRY, M., MILLET, D. et O'YL, W. 1997 - Le gisement acheuléen d'En Jacca - La Sauvegarde à Colomiers (Haute-Garonne) : nouvelles données. Paléo, n 9. pp. 69-99.

CAVAILLÉ A. 1965 - Toulouse ouest. Notice explicative, carte géologique de la France, feuille de Toulouse ouest (1/50 000), 953. Orléans : BRGM,1965, 8 p.

CHALARD P. et ONÉZIME O. 2003 - Rue Bellevue (Fonsorbes). Rapport de diagnostic archéologique, INRAP, Toulouse, $19 \mathrm{p}$.

COLONGE D. à paraître - Économie des matières premières lithiques sur un site acheuléen du piémont pyrénéen : Lanne-Darré à Uglas (Hautes-Pyrénées). Congrès du C.T.H.S., Toulouse, 9 - 13 avril 2001.

COLONGE D. 2001 - L'Acheuléen du plateau de Lannemezan (Hautes-Pyrénées) à travers les exemples de sites des Canaux d'Irrigation des Coteaux de Gascogne. Mémoire de DEA à l'Université de Toulouse II le Mirail, 2001,110 p.

COLONGE D., ARNOUX T., BRUXELLES L., CHALARD P., ONÉZIME O. et coll. de GRALL A. et LELOUVIER L.-A. 2003a - Bichou (Montaigut-sur-Save, Haute Garonne). Rapport d'évaluation archéologique, INRAP, Toulouse, $38 \mathrm{p}$.

COLONGE D., BRUXELLES L., CHALARD P., JARRY M. et ONÉZIME O. 2003b - Rominguière (Cornebarieu, Haute-Garonne). Rapport d'évaluation archéologique, INRAP, Toulouse, 25 p.

COLONGE D., DELFOUR G., FONDEVILLE C., JARRY M. et coll. de ARNOUX T., BERTHET A.-L. 2002 Raspide 2 - ZAC Aéroconstellation (Blagnac, 31). Rapport Final d'Opération, INRAP dir G.S.O., S.R.A. de Midi-Pyrénées, $83 \mathrm{p}$.

GAILLARD C. 1982 - L'industrie du Paléolithique inférieur et moyen de la grotte de Coupe-Gorge à Montmaurin (Haute-Garonne). Gallia Préhistoire, t. 25, CNRS, 1982, pp. 79-105.

GENESTE J.-M. et GÉ T. 2001 - Les implantations du Paléolithique moyen dans la vallée de l'Isle (Dordogne, France) d'après les travaux d'archéologie préventive de l'autoroute A 89. Pré-actes du XIVème congrès de l'UISPP, Liège, 2-8 sept. 2001, Université de Liège, p. 111.

GRIMBERT L., JARRY (M.), ARNOUX T. et ONÉZIME O. 2003 - Plaisance-du-Touch. Z.A. de la Ménude (Haute-Garonne, 31). Rapport de diagnostic archéologique, INRAP, Toulouse, 9 p.

HUBSCHMAN, J. 1975a - Modelés et formations quaternaires du terrefort molassique, au sud de Toulouse. In : Le piémont quaternaire de la Garonne (sud-est du bassin Aquitain, piémont central nord-pyrénéen). Bulletin de l'Association Française pour l'Etude du Quaternaire, nº 44-45, Paris :

Université Pierre et Marie Curie, Laboratoire de Géologie I. pp. 125-136. 
HUBSCHMAN, J. 1975b - Les terrasses récentes de la Garonne et leur évolution. In : Le piémont quaternaire de la Garonne (sud-est du bassin Aquitain, piémont central nord-pyrénéen. Bulletin de l'association française pour l'étude du Quaternaire, $n^{\circ} 44-45$, Paris : Université Pierre et Marie Curie, Laboratoire de Géologie I, pp. 137-147.

HUBSCHMAN, J. 1975C - L'évolution de nappes alluviales antérissiennes de la Garonne, dans l'avant-pays molassique. In : Le piémont quaternaire de la Garonne (sud-est du bassin Aquitain, piémont central nord-pyrénéen. Bulletin de l'association française pour l'étude du Quaternaire, n 44-45, Paris : Université Pierre et Marie Curie, Laboratoire de Géologie I, pp. 148-160.

HUBSCHMAN, J. 1975d - Morphogénèse et pédogénèse quaternaires dans le piémont des Pyrénées garonnaises et ariégeoises. Thèse Doct. Etat, Toulouse. Edit. Honoré Champion, Paris. 745 p.

HUBSCHMAN, J. 1975e - Conclusion. Evolution pédo-géochimique et interprétation paléobioclimatique du piémont quaternaire garonnais. In : Le piémont quaternaire de la Garonne (sud-est du bassin Aquitain, piémont central nord-pyrénéen). Bulletin de l'Association Française pour l'Etude du Quaternaire, n 44-45, Paris : Université Pierre et Marie Curie, Laboratoire de Géologie I. p. 209-216

HUBSCHMAN, J. 2000 - Le cadre géographique de la cité de Toulouse. Terrains, milieux et terroirs. In : Tolosa, Nouvelles recherches sur Toulouse et son territoire dans l'Antiquité. Sous la direction de JeanMarie PAILLER. Collection de l'Ecole Française de Rome - 281. pp. 1-39.

HUBSCHMAN J., ICOLE M., JALUT J., REVEL J.-C., TAILLEFER F. et BOURGEAT F. 1980 - Le Quaternaire nord-pyrénéen. In : CHALINE J. (dir.) - Problèmes de stratigraphie quaternaire en France et dans les pays limitrophes. Actes de la table ronde de Dijon, Bulletin de l'association française pour l'étude du Quaternaire, N.S. n 1, pp. 307-311.

ICOLE M. 1973 - Géochimie des altérations dans les nappes d'alluvions du piémont occidental nordpyrénéen. Essai de paléopédologie quaternaire. Thèse Sciences Paris. 348 p.

ICOLE M. 1980 - L'altération au sein des alluvions quaternaires du piémont occidental nordpyrénéen : éléments de corrélations. In : CHALINE J. (dir.) - Problèmes de stratigraphie quaternaire en France et dans les pays limitrophes. Actes de la table ronde de Dijon, Bulletin de l'association française pour l'étude du Quaternaire, N.S. n 1, pp. 312-313.

ICOLE M. et RIEUCAU, L. 1982a - Les limons quaternaires et les dépôts de pente dans le bassin de la Garonne et ses affluents. In : Préhistoire de Midi-Pyrénées. Extraits de La Préhistoire Française, Ed. du CNRS, 1976, Toulouse : CRDP, DRAC de Midi-Pyrénées, pp. 9-12.

ICOLE M. et RIEUCAU, L. 1982b - Les alluvions fluviatiles dans le bassin de la Garonne et ses affluents. In : Préhistoire de Midi-Pyrénées. Extraits de La Préhistoire Française, Ed. du CNRS, 1976, Toulouse : CRDP, DRAC de Midi-Pyrénées, pp. 5-8.

JARRY M. 1994 - Etude techno-typologique du site d'Esclignac à Montmaurin (Haute-Garonne). Mémoire de DEA, Université de Toulouse-le-Mirail et Ecole des Hautes Etudes en Sciences Sociales, 1994, $105 \mathrm{p}$.

JARRY M. et COLONGE D. 2001 - Raspide 2, ZAC Aéroconstellation (Blagnac, Haute Garonne). D.F.S. d'évaluation archéologique, AFAN-GSO, SRA de Midi-Pyrénées, 24 p.

JARRY M., ARNOUX T. et DELFOUR G. 2002 - En Jacca - La Sauvegarde 2. D.F.S. de diagnostic archéologique, AFAN, S.R.A. de Midi-Pyrénées, Toulouse 2002, 34 p.

JARRY M., BERNARD F., BERTRAND F., CHALARD P., COLONGE D., DEBENHAM N., DIOT M.-F., LELOUVIER L.-A., MOURRE V., TIXIER C. et TURQ A. 2001a - Les Bosses 1 (Lamagdelaine, 46) : un 
gisement paléolithique moyen antérieur à l'avant-dernier interglaciaire sur la moyenne terrasse du Lot. D.F.S., AFAN, S.R.A. de Midi-Pyrénées, Toulouse 2001, 249 p.

JARRY M., BERTRAN P., COLONGE D., LELOUVIER L.-A. et MOURRE V. à paraître - Le gisement paléolithique moyen ancien des Bosses à Lamagdelaine (Lot, France). Actes du XIVéme Congrès de l'U.I.S.P.P., Liège, 2-8 sept. 2001, BAR International Series, 10 p.

JARRY M., COLONGE D., GERNIGON K., LELOUVIER L.-A. avec coll. de BERNARD F. et CAPDEVILLE J.-P. 2001b - En Jacca-Puntis (Colomiers, Haute-Garonne). AFAN dir. GSO, SRA de Midi-Pyrénées, Toulouse, 49 p., 29 fig.

JAUBERT J. 1993 - Le gisement paléolithique moyen de Mauran (Haute-Garonne) : technoéconomie des industries lithiques. Bulletin de la Société Préhistorique Française, 93/5, pp. 328-335.

JAUBERT J. 2002 - Le Paléolithique dans le Midi toulousain. In : PAILLER (J.-M.) dir. - Tolosa, nouvelles recherches sur Toulouse et son histoire dans l'Antiquité. Coll. de l'Ecole Française de Rome, $n^{\circ} 281,2002$. pp. 43-52.

JAUBERT J. et SERVELLE C. 1996 - L'Acheuléen du bassin de la Garonne. Etat de la question et implications. In : A. Tuffreau dir. : «l'Acheuléen dans l'Ouest de l'Europe », actes du colloque international de Saint-Riquier (6-10 juin 1989), publ. du C.E.R.P., Université des Sciences et Techniques de Lille, n 4, 1996, pp. 89-106.

MÉROC L. 1961 - Informations archéologiques, circonscription de Toulouse. Haute-Garonne : Colomiers. Gallia Préhistoire, T.4, p. 247-252.

MÉROC L. et PALOUMÉ J. 1958 - Nouvelles fouilles à l'Infernet, commune de Clermont-le-Fort (Haute-Garonne). Bulletin de la Société Méridionale de Spéléologie et de Préhistoire, années 1954-55. 1958, p. 305-328.

MILLET D. 1994 - Vallée de la Garonne (Haute-Garonne et Tarn-et-Garonne). Prospection thématique - opérations interdépartementales. In : Bilan scientifique de la région Midi-Pyrénées 1994. DRAC Midi-Pyrénées, Service régional de l'archéologie, Toulouse. pp. 225-226.

MILLET D. et F. 1996 - Une coupe acheuléenne à En Tole (Rieumes). Archéo en Saves: Archéologie, Histoire et Ethnographie en Gascogne orientale, $\mathrm{n}^{\circ}$ 9, pp. 2-28.

MILLET D. 2001 - Le Paléolithique inférieur en Aquitaine méridionale. Contribution à l'étude typotechnologique du Paléolithique inférieur de l'axe garonnais, de l'Albigeois et du Bas-Armagnac. Thèse de doctorat nouveau régime, Université Toulouse-Le Mirail, 3 t., 647 p, 425 pl.

PINOS A. 1999 - L'Homme de Neandertal dans le Midi-toulousain : approche typologique et technologique de deux séries lithiques issues de stations de plein air. Archéo en Saves: Archéologie, Histoire et Ethnographie en Gascogne orientale, H.S. n 3, 101 p.

PONS F., avec la collaboration de ARNOUX T., BERTRAN P., LAGARRIGUE A. 2001 - ZAC Aéroconstellation, phase 1 - Blagnac (31). DFS de diagnostic archéologique, AFAN, SRA MidiPyrénées, Toulouse, 2001, 112 p.

SERRA D. 1980 - Les industries acheuléennes de la grotte de la Terrasse, Montmaurin (Haute-Garonne) et inventaire de l'industrie moustérienne du Putois, Montmaurin. Université de Provence, Marseille, Travaux de Paléontologie Humaine et Préhistorique, $n^{\circ} 4,1980,349$ p.

TAVOSO A. 1986 - Le Paléolithique inférieur et moyen du Haut-Languedoc. Gisements des terrasses alluviales du Tarn, du Dadou, de l'Agout, du Sor et du Fresquel, Université de Provence, Ed. du Laboratoire de Paléontologie Humaine et de Préhistoire, Etudes Quaternaires, 5, (1978), 1986, $404 \mathrm{p}$. 
VÉZIAN, J. 1991. - Les stations des premières industries de la Croix d'En Mauran et du Moulin des Graves, à Aiguesvives (Haute-Garonne). Bulletin de la Société Méridionale de Spéléologie et de

Préhistoire, t 31. 1991, pp. 17-32

\section{RÉSUMÉS}

Depuis quelques années, les opérations d'archéologie préventive menées en Midi toulousain ont permis de réactualiser certaines données relatives à la géomorphologie des sites paléolithiques inférieur et moyen. Ainsi, plusieurs contextes peuvent être considérés comme plus favorables que d'autres pour la conservation du matériel. Par ailleurs, les résultats préliminaires d'études typo-technologiques des industries mises au jour récemment, offrent de nouvelles perspectives de recherche pour la constitution d'un cadre chrono-culturel plus précis.

In the last years, preventive archeology projects conducted in the Midi toulousain Region (southern France) allowed to update data concerning the geomorphology of Early and Middle Palaeolithic sites. It became apparent that certain contexts can be considered as more favorable to vestige conservation than others. Besides, preliminary results of typo-technological studies of recently discovered artifacts offer new research perspectives for the definition of a more precise chrono-cultural frame.

\section{INDEX}

Mots-clés : Midi toulousain, géomorphologie, potentialités, Paléolithique inférieur et moyen, archéologie préventive

Keywords : Midi toulousain, geomorphology, potentialities, Early and Middle Paleolithic, preventive archaeology.

\section{AUTEURS}

\section{BRUXELLES}

INRAP et résidence Constance, 31 route de Lattes 34470 Pérols.

\section{A.-L. BERTHET}

INRAP et Le Bourg 33220 Fougueyrolles.

\section{P. CHALARD}

INRAP, centre archéologique, Z.A. des Champs Pinsons, 13 rue du négoce 31650 St.-Orens-deGameville et UMR 5608/UTAH Toulouse.

\section{COLONGE}

INRAP, centre archéologique, espace 10, Bât. B, 17 rue Albin Haller 86000 Poitiers et UMR 5608/ UTAH Toulouse.

\section{G. DELFOUR}

INRAP et Le Vauriat 63230 Saint-Ours. 


\section{JARRY}

INRAP, centre archéologique, Z.A. des Champs Pinsons, 13 rue du négoce 31650 St.-Orens-deGameville et UMR 5608/UTAH Toulouse.

\section{L.-A. LELOUVIER}

INRAP, centre archéologique, Z.A. des Champs Pinsons, 13 rue du négoce 31650 St.-Orens-deGameville.

\section{T. ARNOUX}

INRAP, centre archéologique, Z.A. des Champs Pinsons, 13 rue du négoce 31650 St.-Orens-deGameville.

\section{O. ONÉZIME}

INRAP, centre archéologique, Z.A. des Champs Pinsons, 13 rue du négoce 31650 St.-Orens-deGameville. 